\title{
Circuit
}

Musiques contemporaines

\section{André Villeneuve, Ouvrier d'harmonies, Sainte-Foy, Presses de l’Université du Québec, 2010, 312 p.}

\section{Simon Bertrand}

Volume 21, numéro 1, 2011

Du spirituel dans l'art?

URI : https://id.erudit.org/iderudit/1001168ar

DOI : https://doi.org/10.7202/1001168ar

Aller au sommaire du numéro

Éditeur(s)

Les Presses de l’Université de Montréal

ISSN

1183-1693 (imprimé)

1488-9692 (numérique)

Découvrir la revue

Citer ce compte rendu

Bertrand, S. (2011). Compte rendu de [André Villeneuve, Ouvrier d'harmonies, Sainte-Foy, Presses de l'Université du Québec, 2010, 312 p.] Circuit, 21(1), 93-96. https://doi.org/10.7202/1001168ar

Ce document est protégé par la loi sur le droit d'auteur. L'utilisation des services d’Érudit (y compris la reproduction) est assujettie à sa politique d'utilisation que vous pouvez consulter en ligne.

https://apropos.erudit.org/fr/usagers/politique-dutilisation/
Cet article est diffusé et préservé par Érudit.

Érudit est un consortium interuniversitaire sans but lucratif composé de l’Université de Montréal, l’Université Laval et l’Université du Québec à Montréal. Il a pour mission la promotion et la valorisation de la recherche. https://www.erudit.org/fr/ 


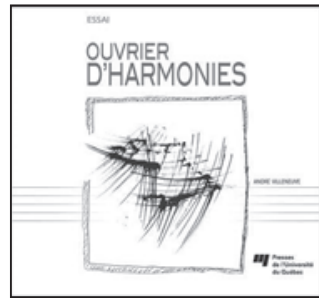

André Villeneuve

Ouvrier d'harmonies

Sainte-Foy, Presses de l'Université

du Québec, 2010, 312 p.

Compte rendu de Simon Bertrand

Il ne faut pas s'attendre ici à des «leçons» d'harmonie au sens académique du terme ou à un ouvrage didactique portant sur ce paramètre musical. Mais au terme d'un siècle où, de Claude Debussy à György Ligeti, les notions de verticalité et d'horizontalité en musique ont considérablement évolué, englobant au passage les harmonies dites «non fonctionnelles», les accords spectraux et la micro-polyphonie, une réflexion plus globale comme celle à laquelle se livre André Villeneuve, compositeur né en 1956 et professeur au département de musique de l'Université de Québec à Montréal (UQAM), nous semble tout à fait pertinente, voire nécessaire. Bien plus qu'un simple paramètre musical donc, l'harmonie dont il est question ici est une sorte d'idéal, l'objet principal d'une quête à la fois poïétique et éthique: une recherche de la conciliation dans la distinction, et entre un soi-même et l'autre. L'harmonie y semble autant l'objet principal d'une recherche esthétique que, d'une certaine manière, d'une forme de quête spirituelle. C'est notamment dans ce sens que cet ouvrage est empreint de l'attitude littéraire de Claude Ballif (1924-2004), compositeur français dont Villeneuve fut l'élève, et dont des livres comme Voyage de mon oreille et Économie musicale emploient un langage métaphorique, à mi-chemin entre la philosophie et une sorte d'introspection sur la genèse de ses œuvres, à l'instar de l'approche de l'auteur d'Ouvrier d'harmonies.

Bien que figurent à la fin de l'ouvrage quelques analyses d'extraits de Webern et de Debussy, Villeneuve se livre pour l'essentiel à des réflexions sur les stratégies poétiques du compositeur, où l'harmonie, «désert fertile que j’interroge ${ }^{2}$ ", y est une sorte de quête, de chemin vers l'œuvre. En douze chapitres de longueur variable, l'auteur illumine son sujet de différents angles. Dès le premier chapitre, intitulé « Nuances et répétitions autour d'un énoncé principal», il développe une réflexion sur l'ensemble de ce qu'il nomme le "parcours de l'ouvrier", un parcours reparti en trois moments distincts: l'idée, le tracé et l'œuvre. Comme il écrit: «Cet ensemble, idéetracé-œuvre, / Est modelé sur nos représentations de ce qu'est cet ensemble. / Un ciel commun à tous. Chacun y fera son parcours» (p. 3). Le but de ce parcours se trouve dans la conciliation entre ces trois moments: «On peut
1. Voyage de mon oreille, Paris, UGE, 1979, et Économie musicale: souhaits entre symbols, Paris, Méridiens-Klincksieck, 1988.

2. André Villeneuve, "Harmonies. Déserts fertiles que j'interroge", Circuit, vol. 10, nº 1 (1999), p. 63-70. 
3. Danick Trottier, "Entrevue avec André Villeneuve sur l'enjeu de l'identité dans la poḯtique musicale", Circuit, vol. 15, $\mathrm{n}^{\circ} 2$ (2005), p. 86. exprimer cette conciliation / comme un désir de cohérence (cohaerens, « rattaché à») / Entre l'abstraction originelle d'une idée et l'œuvre, sa concrétisation. / [...] On peut désigner cette conciliation recherchée par harmonie» (p. 5). Néanmoins, Villeneuve se garde bien de décréter quelque procédé compositionnel ou concept spécifique que ce soit, et reste, d'abord et avant tout, un interrogateur de l'ouvrier-compositeur : «La part du calcul? La part de l'intuition? / Pourquoi les distinguer?» (p. 12).

Dans le chapitre 2, «Interrogations de l'ouvrier », l'auteur conçoit l'harmonie comme processus poḯtique global: «Harmonie : le concept est tout entier livré à son entendement. / Harmonie relève de ses représentations saisies / Sur une multitude de représentations possibles » (p. 17). On y trouve aussi une belle métaphore, éloge de l'harmonie en tant que nuage, source et ressource continuelle du discours musical :

Chacun y fera son parcours selon ses représentations. / Mais la plus belle représentation de cela est, peut-être, celle du nuage: / A devenant A devenant A / A-A-A / Ce nuage, devenant cet autre, devenant cet autre, jamais le même. (p. 18)

Plus loin, Villeneuve s'exprime plus concrètement: «L'accord. / Il instruit./ Par autorité du vertical et de l'horizontal, / [...] Intervalle comme mesure du sonore ou résonant particulier, / Couleur ou panorama d'inventions, pôle ou entité complexe, / L'accord est cohérence» (p. 25). Ces multiples représentations de l'harmonie approchent même par moments une certaine forme de mysticisme: «La Foi : / Il me semble que cela est une / harmonie de conciliation entre soi et ce qui est au-dessus de soi» (p. 29). Cela n'est pas sans nous rappeler les propos de Villeneuve dans un entretien de 1999 où l'on retrouve cette même représentation de l'harmonie comme quête spirituelle :

Pour certains, cela est la Foi : harmonie de conciliation / entre soi et ce qui est au-dessus de soi; pour / d'autres, l'éthique: harmonie de conciliation entre soi / et ses engagements; pour certains, un accord suffira, / pour d'autres, le pinceau ou le poème 3 ...

Dans le chapitre 3, "L'ouvrier interroge sa position temporelle dans sa quête de l'idée », une nuance importante est cependant évoquée sur le plan de la temporalité: «Ainsi, voilà peut-être deux harmonies de tensions bien différentes l'une de l'autre: / A, l'ouvrier en quête de B, l'idée / ou / A B: L’ouvrier déjà dans le temps de $\mathrm{B}$ » (p. 36). Pour sa part, le chapitre 4 développe la notion de contrainte, tout comme d'autres problématiques propres au chemin vers l'œuvre de l'ouvrier: «Une idée est en soi une contrainte. / Et sans contrainte nous ne sommes rien. / L'outil des limitations et des 
nécessités est à notre portée» (p. 41). De même, dans le cinquième chapitre, «L'ouvrier interroge le temps concret de l'œuvre et du signe », nous sommes toujours en plein cœur des contraintes et des choix qui sont le propre du travail de l'ouvrier :

Euvrer sur une idée est aussi œuvrer sur ce que l'on peut en suggérer dans la mesure où une portion de ses possibilités est élue, saisie par les signes et, par la nuance des interprétations, projetée comme un tout de ses possibilités. L'idée: un infini de possibilités. (p. 47)

Dans le chapitre 6, on trouve un élément de réflexion sur la notion de contraste par rapport à un matériau plus ou moins minimal qui nous semble, à la croisée des chemins entre la nouvelle complexité et le minimalisme, des plus valables. L'auteur étale une échelle de possibilités de relations entre un matériau minimal ou diversifié et la notion essentielle de contraste à l'intérieur du discours musical: «Un matériau diversifié peut être peu contrasté / Un matériau diversifié peut être très contrasté / Un matériau minimal peut être peu contrasté / Un matériau minimal peut être très contrasté » (p. 52 ). Dans le chapitre 7 , il étend ses possibilités jusqu'au choix entre le rationnel et l'irrationnel. Tout le paradoxe de l'acte créateur y est mis à nu: «L'acte est volontaire (le labeur d'œuvrer), / mais la volonté qui l'anime est irrationnelle (ou ne l'est-elle pas?). / Participe-t-elle, peut-être, d'une autre volonté, plus irrationnelle encore: / la volonté de se signifier au monde, d'y prendre la parole?» (p. 57).

Quant au bref chapitre 8, qui a pour titre «Le “je" de l'ouvrier ", l'ouvrier du titre prend une distance par rapport au long périple qui l'amène à l'œuvre, se demandant: «l'imposition des limites des signes de l'écriture permettent-ils / à l'Ouvrier de clamer de manière retentissante ce "je" ? " (p. 62). Le chapitre 9 est très substantiel. Intitulé «L'ouvrier interroge la répétition », il aborde la dynamique entre le signe et la répétition, et la situe en tant qu'outil essentiel du faire de l'ouvrier. Villeneuve résume cette dynamique succinctement dans le passage suivant: «Le signe dans la répétition. / Il est un outil de nuances parmi d'autres outils de nuances. / Le considérer, en matière de composition musicale, / Comme une instrumentation du sonore » (p. 73). Dans le chapitre 10, Villeneuve s'interroge sur la valeur que l'ouvrier accorde au creatio : «L'Ouvrier lui confère-t-il une valeur d'artisanat (crescere, "crô̂tre, faire pousser, faire grandir»)? / Y rattache-t-il une conception plus large, une valeur spirituelle (creator)? / Ou selon «sa propre symbolique personnelle» (Ballif) ... naturellement éveillé par la conscience de sa position temporelle, à toutes ces représentations? » (p. 85). 
4. Voir Serge Provost, "Gilles Tremblay: éléments d'une poétique musicale vus sous le prisme de Réseaux, Fleuves et Solstices", Circuit, vol. 20, no 3 (2010), p. 105-120.

5. Chez Ballif, le référentiel est un certain assemblage d'intervalles qui n'est pas neccésairement une série mais qui, un peu comme dans la notion de talea et de color de l'Ars nova du XIVe siècle, tiendrait lieu de color.
C'est dans le chapitre 11, nommé « Nuances de nos représentations du travail d'harmonie de l'ouvrier ", que Villeneuve suggère, en guise de point culminant précédant la présentation des exemples analytiques, une sorte de lexique pour une poḯtique de l'harmonie, un répertoire qui pourrait bien servir de référence pour une recherche sémiologique sur ce paramètre musical. En effet, l'auteur y offre de brèves définitions d'une catégorisation hautement originale de divers types d'harmonie, une typologie que Villeneuve avait déjà amorcée dans son article qui a paru dans le vol. 10, nº 2 (1999) de Circuit: «Harmonies D’euphonies, Synesthésiques, Phonétiques, Hiératiques, Polyphoniques, De points pivotants, D'assises, D'instants variables, D'inflexions, D'émergences, In absentia» (p. 95). C'est à la fin de ce chapitre que l'on trouve quelques propositions analytiques sur des extraits d'œuvres de Webern et de Debussy, à usage sans doute pédagogique, qui servent d'illustration des propos élaborés dans les autres chapitres. On y retrouve également quelques extraits de partitions correspondants en annexe, ainsi qu'un extrait de Fleuves de Gilles Tremblay4. Bien qu'il ne s'agisse que d'analyses fragmentaires, il faut néanmoins souligner que le choix de ces œuvres est tout à fait apte à illustrer sa conception de l'harmonie en tant que processus poïétique global. Il est à noter au passage également que l'approche analytique de Villeneuve n'est pas sans rappeler celle de Claude Ballif, en particulier de sa notion de «référentiel5».

Enfin dans le douzième et dernier chapitre, intitulé «Où il s'agit de donner suite à l'énoncé initial », sorte d'épilogue, Villeneuve jette un long regard sur l'ensemble des réflexions du livre. Il y aborde la question du silence d'une manière qui laisse peut-être transparaître une certaine forme de tristesse existentielle: «Peut-être que le poids d'un certain silence / Est la raison pour laquelle il fait sonner / Peut-être que le poids d'un certain silence / Est le point d'origine de ses efforts d'harmonies» (p. 116). Si le but de ce livre était d'essayer de faire un nouveau type d'ouvrage pédagogique non didactique, sorte de compagnon de parcours de l'ouvrier-compositeur à la recherche de sa propre représentation de l'harmonie, alors nous pouvons saluer un ouvrage qui, malgré son côté répétitif (vraisemblablement volontaire), ouvrira, souhaitons-le, la voie à une nouvelle forme de discours sur la composition qui oserait enfin aborder les processus de création des compositeurs, plutôt que de se concentrer sur de simples recettes de cuisine. En ce sens, l'ouvrage d'André Villeneuve est plus que bienvenu. 